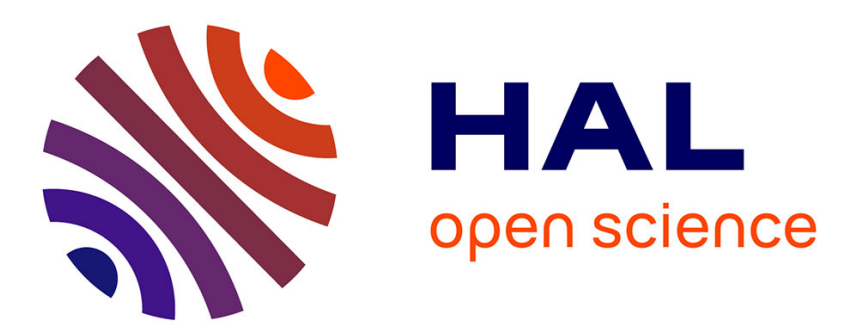

\title{
Les sentiers d'interprétation glaciaire: des outils de valorisation différenciée des glaciers et de leur territoire
}

\author{
Nathalie Cayla
}

\section{To cite this version:}

Nathalie Cayla. Les sentiers d'interprétation glaciaire: des outils de valorisation différenciée des glaciers et de leur territoire. Neige et glace de montagne: Reconstitution, dynamiques, pratique., Collection EDYTEM - Cahiers de Géographie, nº, pp.221-232, 2009. halsde-00391967

\section{HAL Id: halsde-00391967 \\ https://hal.science/halsde-00391967}

Submitted on 30 Mar 2011

HAL is a multi-disciplinary open access archive for the deposit and dissemination of scientific research documents, whether they are published or not. The documents may come from teaching and research institutions in France or abroad, or from public or private research centers.
L'archive ouverte pluridisciplinaire HAL, est destinée au dépôt et à la diffusion de documents scientifiques de niveau recherche, publiés ou non, émanant des établissements d'enseignement et de recherche français ou étrangers, des laboratoires publics ou privés. 


\section{Collection EDYTEM}

Numéro 8 - Année 2009

Cahiers de

Géographie

\section{NEIGE et GLACE de MONTAGNE}

Reconstitution, dynamique, pratiques

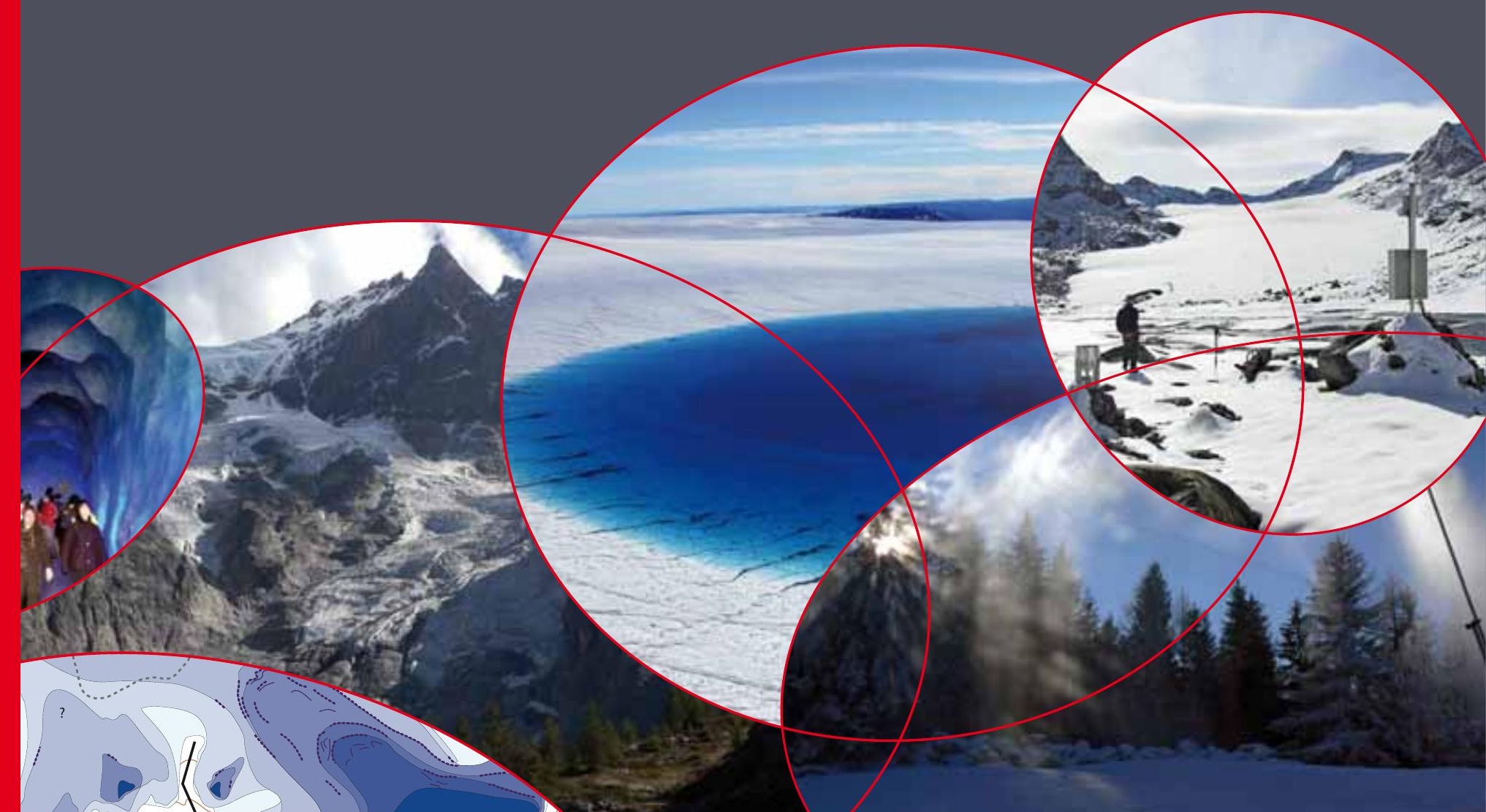





\title{
LES SENTIERS D'INTERPRÉTATION GLACIAIRE
}

\section{DES OUTILS DE VALORISATION DIFFÉRENCIÉE DES GLACIERS ET DE LEUR TERRITOIRE}

\author{
INTERPRETATIVE GLACIER TRAILS: \\ TOOLS FOR THE VALORISATION OF GLACIERS AND HIGH APINE VALLEYS \\ NATHALIE CAYLA \\ Laboratoire EDYTEM, Université de Savoie /CNRS, Campus scientifique, F 73376 Le Bourget-du-Lac cedex. \\ Contact : nathalie.cayla@univ-savoie.fr
}

\begin{abstract}
RÉSUMÉ
Les glaciers peuvent se révéler de remarquables géosites, dont l'importance sociétale se renforce avec le réchauffement global qui menace leur existence dans les Alpes. Parmi l'ensemble de l'offre en matière de sentier d'interprétation au cœur du massif alpin, 30 réalisations permettent de découvrir autant de vallées glaciaires, abritant toujours un glacier. L'analyse de la répartition spatiale de ces sentiers montre que des pays comme l'Autriche et l'Italie sont particulièrement actifs dans la création de ce type d'offre écotouristique qui allie des pratiques sportives de pleine nature respectueuses de l'environnement à la découverte de celui-ci. Les jeux d'acteurs à l'origine de ces créations permettent d'établir une typologie expliquant en partie la répartition observée. L'analyse qualitative des sentiers eux-mêmes ainsi que des outils de médiation produits afin de guider le randonneur nous permettra de mieux cerner les objectifs poursuivis par les concepteurs et donc les messages qu'ils cherchent à faire passer auprès du public.
\end{abstract}

Mots-CLÉS : PATRIMOINE GÉOMORPHOLOGIQUE, GÉOSITE GLACIAIRE, SENTIER D 'INTERPRETATION, VULGARISATION SCIENTIFIQUE.

\begin{abstract}
The glaciers can be remarkable geosites, whose societal importance strengthen with global warming which threatens their existence in the Alps. Among the whole offer of interpretative trails in the alpine ark, 30 achievements allows to discover as many glacial valleys, sheltering still today glaciers. The spacial analysis of these trails shows that countries like Austria and Italy are particularly actives in the creation of this type of ecotouristic tools which combine outdoor practices respectful of the environment in order to discovered this one. The sets of actors at the origin of these creations make it possible to establish a typology partly explaining the distribution observed. The qualitative analysis of the paths themselves as of the tools for mediation produced in order to guide the hiker will enable us to better determine the aims of creators and thus messages which they seek to transmit to the public.
\end{abstract}

KEYWORDS: GEOMORPHOLOGICAL HERITAGE, GLACIER GEOSITE, GLACIER DIDACTIC TRAIL, SCIENTIFIC POPULARIZATION. 


\section{INTRODUCTION}

Les glaciers sont des objets géomorphologiques à haute valeur patrimoniale en raison de leur intérêt majeur dans différents domaines (Reynard, 2004) :

- intérêt scientifique, qui a donné naissance à une discipline particulière : la glaciologie et plus largement, dans le cadre de la géomorphologie, l'étude de l'héritage glaciaire qui les entoure ainsi que des séquences paraglaciaires (Mercier, 2007) au sein desquelles ils s'insèrent ;

- intérêt esthétique, les paysages glaciaires, évoquant, dans nos sociétés une nature primitive et préservée, jusqu'à symboliser une certaine pureté exploitée, parfois avec excès, dans les messages publicitaires ;

- intérêt économique, car pourvoyeurs de ressources : la glace, l'eau, l'énergie mais aussi l'ensemble des pratiques touristiques qui y sont liées ;

- intérêt culturel, hommes et glaciers ayant au cœur des Alpes une histoire commune déjà bien ancienne, ponctuée d'événements recensés par la géocyndinique ;

- intérêt pédagogique, les glaciers et leur environnement permettant des observations de terrain particulièrement démonstratives dans la perspective d'une pratique d'éducation non-formelle.

Le réchauffement global actuel n'a fait qu'amplifier cette importance sociétale déjà reconnue par des labellisations prestigieuses : le glacier d'Aletsch est devenu patrimoine mondial de l'UNESCO en 2001, le parc naturel régional Adamello Brenta étant lui devenu European \& UNESCO Global Geopark en 2008.

A partir des années 70, le maillage des territoires par des espaces naturels protégés a fait émerger la nécessité d'y développer des formes d'éducation à l'environnement, qui se sont concrétisées par la création d'écomusées ou de centres d'interprétation. Parallèlement, ce courant de l'interprétation, déve- loppé au cœur des parcs américains dans le cadre des sorties accompagnées (Tilden, 1957), a gagné les pratiques de découverte de terrain et conduit à l'apparition des sentiers de découverte qui, depuis, ne cessent de se développer (Smiraglia, 2005). En effet, la randonnée est une des activités phare des touristes ayant choisi une destination où la nature occupe un place prépondérante puisque $46 \%$ des français disent la pratiquer (source INSEE, 2000). C'est pourquoi, allier activités de pleine nature et découverte de l'environnement sont des objectifs clés de l'écotourisme Les Alpes n'ont pas échappé à l'émergence de ces aménagements et les sentiers d'interprétation consacrés au thème des glaciers ont pris leur place au sein de cette offre (Cayla, 2009).

Après avoir défini et inventorié la liste des sentiers sur laquelle se fonde notre étude, nous tenterons, par l'analyse quantitative et qualitative de cette offre, de mieux comprendre les objectifs poursuivis par les acteurs ayant participés à la création de ce type d'aménagement. L'analyse quantitative du corpus des sentiers retenus nous permettra de réaliser une typologie rendant compte en partie de la répartition spatiale observée et des jeux d'acteurs en présence. Les choix sociétaux qui ont conduit à la création de cette offre touristique seront interrogés au regard des différences que l'étude comparatives des cinq pays de l'arc alpin entrant dans notre inventaire aura mis en perspective. Si les enjeux liés à l'éducation, à l'environnement, au transfert de résultats de recherche ainsi qu'à la sensibilisation aux impacts du réchauffement climatique gouvernent une majeure partie des réalisations, d'autres enjeux seront aussi mis en évidence. L'étude du contenu didactique des supports de médiation réalisés afin d'accompagner le randonneur permettra ainsi de montrer combien ces outils peuvent participer à la diffusion des connaissances géomorphologiques auprès du grand public.

\section{I - Panorama de la diversité de l'offre EN MATIÈRE DE SENTIERS D'INTERPRÉTATION GLACIOLOGIQUE}

\section{1 - Le développement d'une offre... jusqu'à saturation?}

Les sentiers balisés, dans le but de transmettre des informations scientifiques concernant le milieu parcouru par le visiteur, sont qualifiés tantôt de sentiers à thème, de découverte, voire didactique, ou de parcours d'interprétation. Nous choisirons ici d'exclure le qualificatif de "didactique» que Jean-Pierre Astolfi (2005) attribue plus précisément à tout ce qui relève de la réflexion conduite en amont de la réalisation de cet outil de transmission du savoir qu'est le sentier à thème. Nous nous interrogerons, toutefois, dans la suite de cet article, sur les transpositions didactiques effectuées lors de l'élaboration des vecteurs de médiation, fascicules d'accompagnements ou panneaux posés le long du sentier. Les premières expériences de sentier didactique à thème naturaliste ont vu le jour en Angleterre, où la ville de Rochdale (Lancashire) proposa le premier «geotrail» urbain en 1881. En 1925, les Etats-Unis créèrent cet outil d'interprétation dans le Parc National Palisade Interstate sur la côte Est, et en 1930, l'Allemagne en développa un dans la forêt de Bredower, au cœur du Land de Brandebourg (Hose, 2008). A partir des années 1960, cet outil de valorisation des territoires se généralise. Aujourd'hui, l'Allemagne possède plus de 1080 sentiers à thèmes dont la majeure partie se situe en Bavière où l'on en compte un pour 194 km². 
Les principaux thèmes abordés sont : la forêt $(35 \%)$, les biotopes $(28 \%)$, les paysages $(18 \%)$, la géologie et la géomorphologie $(7 \%)$, l'histoire $(5,5 \%)$ et des thèmes multiples pour les $6,5 \%$ restant (Kestler, 2005). En Autriche, il en existe plus de 700 dont $9 \%$ portent sur la géologie (Eder, 2007). Dans ce pays, un portail internet a été créé par le ministère de l'environnement donnant accès, par région et par thème, à une base de données de 350 de ces sentiers (http://lehrpfade.lebenministerium.at). Quant à la Suisse, Suisse tourisme recensait déjà 300 sentiers à thème en 1999 (RohnBrossard, 2006).

Le premier sentier d'interprétation sur le thème de la géologie a vu le jour dans les Alpes au milieu des années 1970, au cœur des Dolomites, à l'initiative du musée communal de Predazzo et du géologue Elio Sommavila, professeur à l'Université de Ferrare, en partenariat avec l'office de développement touristique du Val di Fiemme ainsi que la société de remontées mécaniques locale. Le sentier géologique des Dos Capèl permet la découverte de l'histoire du massif du Latemar depuis 270 millions d'années, époque du dépôt des importantes séries volcaniques qui en constituent la base (Dellantinio, 2007).

Peu de temps après, en 1978, le Club Alpin Autrichien, l'OeAV (Osterreichischer Alpenverein), qui militait alors activement pour la création du Parc National des Hohe Tauern créa le premier sentier glaciologique des Alpes : le sentier glaciaire d'Innergschlöß. Celui-ci conduit le randonneur depuis le village de Matrei, dans le land de Salzburg, jusqu'à la langue terminale du glacier du Schlatenkees, dans le massif du Grossvenediger, l'un des glaciers alpin qui connaît actuellement le plus fort recul.

Après une période de progression lente, cette offre touristique a connu une croissance importante dans les années 2000 et atteint actuellement une certaine forme de saturation avec 30 sentiers recensés en 2008 (figure 1).

En effet, ces parcours d'interprétation ne représentent qu'à peine $20 \%$ de l'ensemble des sentiers abordant les thèmes de la géologie (environ 130 dans les Alpes) qui eux même sont minoritaires par rapport à l'ensemble des sentiers à thème au sein de ce même espace alpin. D'où une multiplication des panneaux, même si ce ne

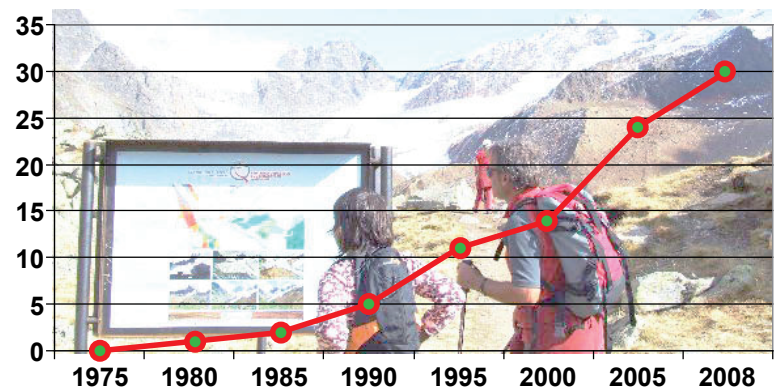

Figure 1 - Evolution quantitative de l'offre de sentiers sur le thème des glaciers dans les Alpes. sont parfois que de simples bornes, dommageable aux paysages que l'on cherche à faire apprécier.

Les réalisations permettant de découvrir la géomorphologie héritée de la dernière période glaciaire, comme le sentier des Chavands dans la vallée de Chamonix, ou bien le sentier Antonio Stoppani dans le Trentin, ont volontairement été exclues de cette étude qui se limite aux parcours de découverte atteignant des glaciers. Nous avons choisi, par contre, d'y intégrer des itinéraires s'intéressant aux glaciers rocheux bien que ces géomorphosites n'aient de glacier que le nom.

En réaction à cette artificialisation de ces territoires remarquables (photo 1), on observe, depuis quelques temps, l'émergence de nouvelles formes de sentiers à thèmes qui peuvent encore être liés à l'espace physique ou bien n'être que virtuels. Ainsi, l'Ecole polytechnique fédérale de Zürich, s'appuyant sur le sentier glaciologique de Morteratsch, a créé une interface web (http://www.klimaweg.ethz.ch/, consultation janvier 2009) entièrement dédiée à la compréhension de l'impact du réchauffement global. Une autre réalisation, du même type, est en train de voir le jour concernant le glacier Marlet dans la vallée de Solda au cœur du parc national de Stelvio. Elle concernera, outre des aspects culturels locaux, des données récentes concernant la dendrochronologie (Caravaglia, 2008).

On voit aussi apparaître des sentiers développés à partir de guide GPS numérique multimédia tel que celui en réflexion dans le parc naturel régional Veglia Devero. Ces réalisations ne se matérialisent par aucune empreinte sur le territoire puisque l'itinéraire tout comme les informations didactiques sont embarqués dans l'interface GPS-multimédia.

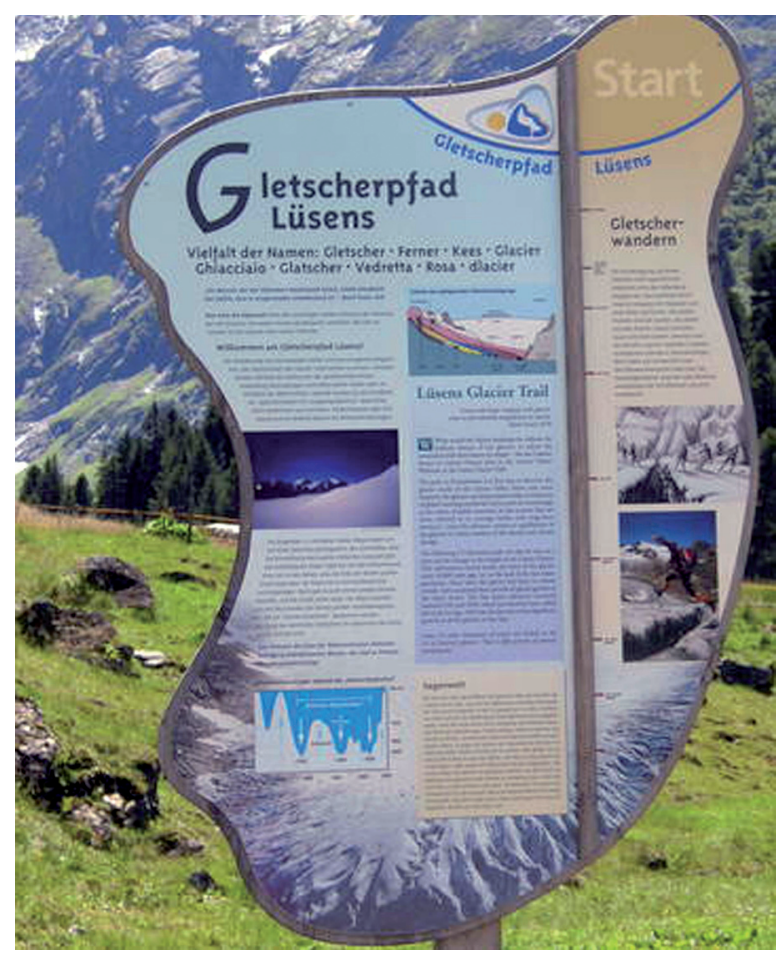

Photo 1 - Panneau de départ du sentier glaciaire de Lüsens (source : office de tourisme de Lüsens - Autriche). 


\section{2 - Des parties prenantes à l'origine d'une répartition inégale de l'offre}

Il existe plus de 5000 glaciers alpins mais $90 \%$ d'entre eux ne dépassent pas $1 \mathrm{~km}^{2}$. Les 30 sentiers inventoriés ici, mettent en valeur, pour la plupart, certains des glaciers les plus renommés des Alpes mais aussi d'autres moins spectaculaires (figure 2).

Afin de faciliter l'analyse de cette répartition spatiale, il nous a semblé nécessaire de mettre en perspective ces premiers résultats avec l'offre quantitative globale des sentiers à thème géologique proposé par chacun des pays, mais aussi de les comparer avec la surface occupée par ces pays dans la surface des Alpes (chiffres de la convention alpine), la surface glaciaire présente dans le pays (Pirocchi, 1998), ainsi que la superficie occupée par les Alpes dans chacun de ces pays (figure 3).

L'Autriche et l'Italie, qui ont des surfaces glaciaires comparables, présentent un nombre de sentiers glaciologiques important avec respectivement 11 et 8 réalisations, ce qui reflète leur place dans l'offre de sentiers à thème géologique dans les Alpes puisque l'Autriche possède plus de 35 sentiers de ce type et l'Italie 27.
Nbre de sentiers d'interprétation sur les glaciers

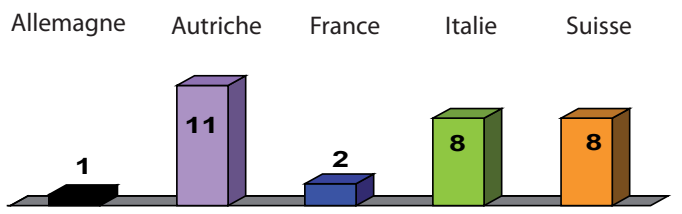

Nbre total de sentiers d'interprétation sur la géologie et la géomorphologie
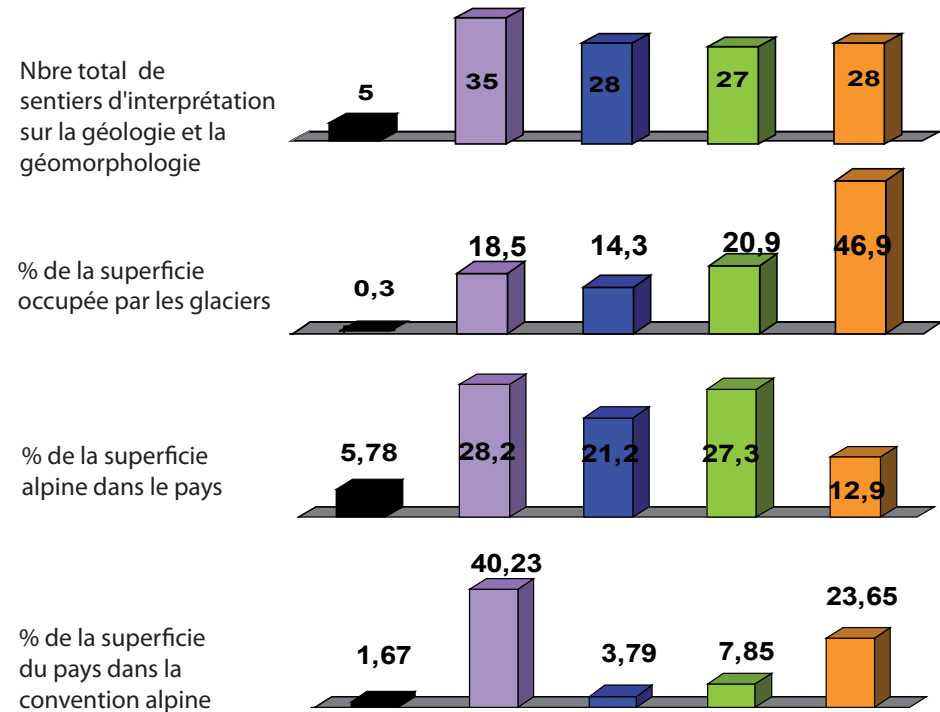

Figure 3 - Analyse comparative de la superficie alpine et glaciaire par rapport à l'offre de sentiers à thème géologique dont les sentiers glaciodidactiques.

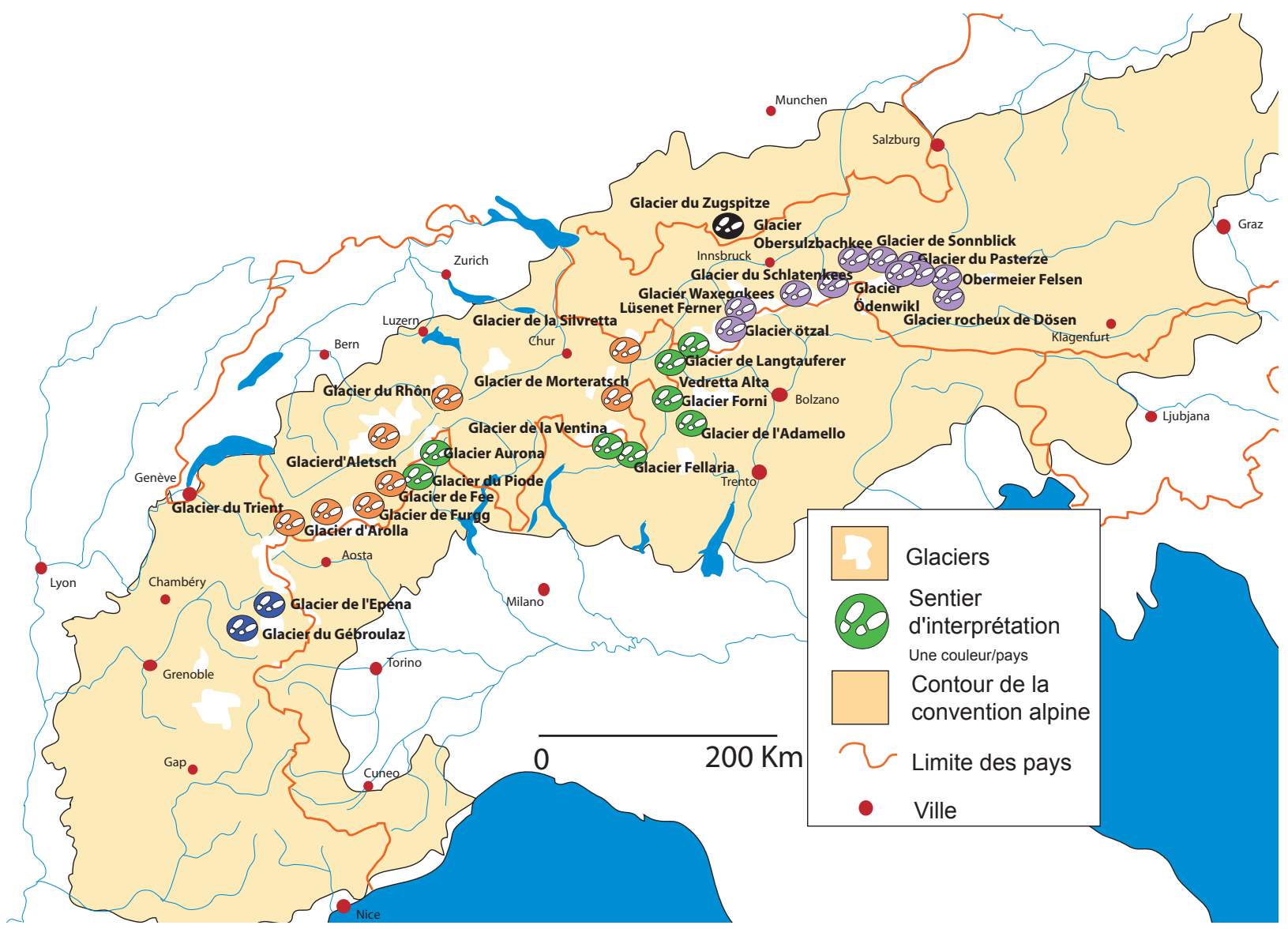

Figure 2 - Répartition des glaciers objets d'une valorisation par un sentier d'interprétation. 
Dans ces deux pays, des organismes ont joué un rôle particulièrement important dans le portage de ces projets : le Club Alpin Autrichien, créé en 1862, fort de 360000 membres pour une population de 8 millions d'habitants (Le Club Alpin Français n'affiche que 82000 adhérents) et le Comité Glaciologique Italien, créé en 1895, à l'initiative du Club Alpin Italien, devenu autonome depuis, dont la principale mission est le suivi scientifique des glaciers du pays. Ces deux organisations non gouvernementales ont été des groupes de pression actifs dans le développement des outils de transfert des connaissances vers un public qui, dans chacun de ces pays, est attaché depuis toujours à ce patrimoine alpin. Rappelons que le parc national du Grand Paradis a vu le jour en 1922 en Italie, suivi en 1935 de celui du Stelvio. Quant à l'Autriche, elle abrite, avec le parc des Hohe Tauern, le plus grand parc national des Alpes. Cette analogie est d'autant plus étonnante que si les Alpes représentent près de $40 \%$ du territoire autrichien, elles ne dépassent pas 7,8\% du territoire italien.

On aurait pu s'attendre à ce que la Suisse, qui possède la surface glaciaire la plus importante de la chaîne, et qui a développé une offre de sentier géologique comparable aux deux pays précédents, occupe une place prépondérante dans l'offre de sentier sur le thème des glaciers. Ce n'est pas le cas, en effet, dans ce pays, même si l'on observe des différences importantes suivant les cantons, chaque sentier est le fruit d'une initiative individuelle, portée par une commune, un centre de recherche voire une section locale du Club Alpin Suisse, sans qu'une mobilisation à une plus grande échelle ait pu conduire à constituer un réseau d'acteurs efficace sur ce thème.

La France, avec ses deux seules réalisations, fait ici figure de dernier de la classe, car même si sa surface glaciaire est réduite, toutes les conditions naturelles sont présentes pour que d'autres glaciers puissent bénéficier de la même mise en valeur. Plusieurs raisons peuvent expliquer ce manque de concrétisation sur le thème des glaciers alors que l'offre de sentiers à thème géologique en France est du même ordre de grandeur que dans les autres pays : le désintérêt des laboratoires de recherche pour ce type d'aménagement qui n'est pas un enjeu dans la carrière d'un chercheur ; la sous-représentation des géomorphologues et des géologues dans les équipes techniques des espaces naturels protégés, d'où le peu d'initiatives sur ces thèmes ; enfin, une demande sociale certainement moins présente que dans les autres pays des Alpes en relation avec la faible empreinte alpine dans notre culture et l'inexorable disparition des réseaux naturalistes. D'où le manque de projets initiés par des communes qui ne perçoivent pas l'intérêt de créer ce type de produit touristique.

Enfin, en Allemagne, l'Université de Bonn, dans le cadre du groupe de recherche géomorphologique du SEDAG (Sediment Cascades in Alpine Geosystems), a développé un itinéraire qui met en valeur la vallée de Reintal conduisant au seul glacier du pays. Cette réalisation est le résultat d'un programme de recherche qui, comme souvent dans ce pays, mais aussi en Autriche, Italie et Suisse, a donné lieu à une valorisation finale auprès du plus large public.

\section{II - OBJECTIFS POURSUIVIS LORS DE LA CRÉATION D'UN SENTIER À THÈME : JEUX D'ACTEURS AU CEUR DES TERRITOIRES}

La mise en place de sentiers didactiques naturalistes relève de trois logiques d'acteurs, non exclusives les unes des autres. Celles des acteurs de la protection des espaces naturels, celles des scientifiques et celles des aménageurs impliqués dans le développement touristique. (figure 4)

Bien que fondamentalement distincts à l'origine, les différents objectifs poursuivis peuvent se croiser selon toutes les combinaisons possibles, une synergie optimale ayant pu être observée, par exemple, dans le cas du sentier glaciaire de l'Obersulzbachtal où de nombreux partenaires ont agi en commun : le parc national des Hohe Tauern, le club alpin autrichien, la commune de Neukirchen, ainsi que le laboratoire de géographie de l'Université de Salzbourg (Slupetsky, 1988). Dans ce cas, en effet, les objectifs poursuivies par les trois catégories d'acteurs ont permis la réalisation d'un sentier particulièrement intéressant dans son contenu. Mais dans quel but chaque acteur investi ce champ du sentier d'interprétation?

\section{1 - L'éducation à l'environnement par les acteurs des espaces naturels protégés}

Un gestionnaire d'espace naturel protégé doit garantir la préservation des ressources naturelles dont il a la charge, tout en assumant un rôle dans l'éducation à l'environnement. Le développement d'un sentier d'interprétation peut répondre à ce double objectif . En effet, pouvant éventuellement faire l'objet de visites sous la conduite d'un garde-moniteur, il permet de mieux accueillir le public, de canaliser ses déplacements et ainsi de préserver le milieu. De plus, le message délivré peut s'appuyer sur un discours naturaliste pouvant aller vers des versions plus environnementalistes abordant alors des thèmes planétaires dont, pour notre cas, le réchauffement global (Arsenault, 2001). La mission de sensibilisation à l'environnement, intrinsèque de ce type de structure, pourra susciter une prise de conscience éco-responsable du randonneur dans le but de le conduire à limiter, durablement, ses émissions 


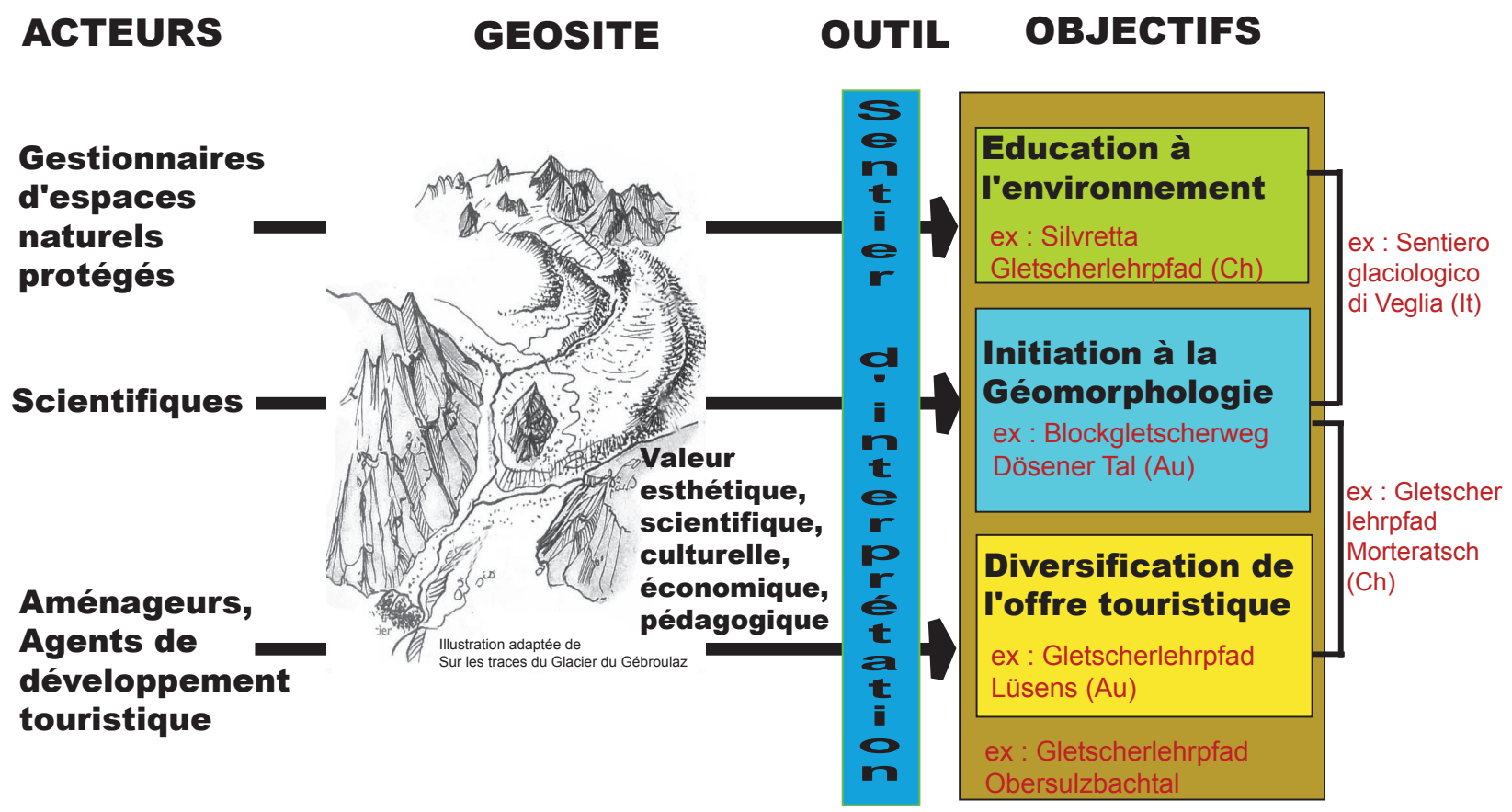

Figure 4 - Jeux d'acteurs à l'origine des sentiers d'interprétation glaciologiques.

de $\mathrm{CO}_{2}$, mais aussi à mieux le former aux choix sociétaux que nos pays seront contraints de prendre dans le futur.

Le parc national des Hohe Tauern, créé dans la décennie 80 , d'une superficie de $1800 \mathrm{~km}^{2}$, possède encore plus de $10 \%$ de sa surface recouverte de glace (soit près de $200 \mathrm{~km}^{2}$ ), ce qui en fait l'espace protégé le plus englacé des Alpes, et, par conséquent, un espace subissant d'importantes modifications paysagères depuis le début $\mathrm{du} \mathrm{XX}^{\mathrm{e}}$ siècle. Les glaciers ont d'ailleurs été un enjeu particulièrement important lors de sa création, car menacés par des aménagements touristiques lourds comme c'est le cas dans d'autres régions du pays. Le Club Alpin Autrichien (OeAV), qui poursuit, entre autres missions, l'étude scientifique du massif alpin, a participé de façon active à la création de la plupart des sentiers glaciaires des espaces protégés autrichiens, soit 9 des 11 sentiers du pays. Il entretient un partenariat principal étroit avec le parc national, ainsi qu'avec d'autres espaces protégés, ce qui explique les nombreuses réalisations observées ( 8 sur les 9 réalisés dans les aires protégées). Dans ces cas, l'OeAV et le parc ont travaillé en synergie avec des scientifiques, spécialistes du domaine, des universités de Graz, de Salzbourg et d'Innsbruck, afin de réaliser des sentiers qui combinent à la fois les résultats des recherche menées sur les glaciers et la nécessaire transposition didactique de ces notions à destination d'un public de passionnés non spécialistes. Chaque parcours est accompagné d'un guide de randonnée d'au minimum une cinquantaine de pages.

Parfois, aussi, un chercheur ou une équipe de recherche prend l'initiative de valoriser auprès du plus grand nombre, par le biais d'un itinéraire de découverte, les résultats de son travail dans le but de mieux faire connaître la géomorphologie en tant que champ disciplinaire (Sellier, 2009).

\section{2 - La diffusion des connaissances scientifiques par les acteurs de la recherche}

Lorsqu'un laboratoire spécialisé dans la géomorphologie ou la glaciologie se lance dans l'aventure de la médiation scientifique, il pénètre sur le territoire du troisième homme, place attribuée par les didacticiens au médiateur scientifique. Les difficultés vont alors surgir afin de bien définir le corpus disciplinaire qui sera retenu, le niveau sémantique qui sera employé, ainsi que les processus didactiques qui seront utilisés : reformulation, analogie et métaphore (Jacobi, 1999)... Le but, ici, n'est pas de produire un discours scientifique destiné à des pairs ou à des étudiants mais un discours adapté à un public non spécialisé occupé à une pratique avant tout de loisir. Il sera donc nécessaire d'adopter, dans cette perspective, les outils de diffusion des connaissances destinés à favoriser la compréhension d'un lieu, ou d'un objet, au travers du thème qui nous occupe ici : les glaciers.

En Italie, l'action conjointe du comité glaciologique national et de sa section lombarde, assistés par les scientifiques du département des sciences de la Terre «Ardito Desio» de l'Université de Milan, a permis, en quelques années, grâce au soutien des autorités territoriales locales, de créer en 1992 le sentier Vittorio Sella (alpiniste et explorateur) sur le glacier de la Ventina, 
puis en 1995 le sentier du centenaire sur le glacier Forni, et enfin, un an plus tard, le sentier Luigi Marson (géographe) sur le glacier de Fellaria. Ces trois réalisations font une large place aux campagnes systématiques de relevés glaciologiques menées depuis la fin du XIX ${ }^{\mathrm{e}}$ siècle. Elles sont principalement axés sur le glacier support du sentier, illustrant les propos de nombreuses photos prises lors des différentes campagnes mais aussi des cartes géomorphologiques, et font une large part aux différents types de recherches scientifiques menées sur ces glaciers : bilan de masse, dendrochronologie... Parfois, la création du sentier est réfléchie au cœur même du travail de recherche ; ainsi, Michaela Nutz, de l'Université de Graz en Autriche a effectué sa thèse sur la mise en place du premier sentier autrichien concernant les glaciers rocheux, le permafrost et le pergélisol (Nutz, 2003). Les glaciers rocheux, longtemps considérés comme des glaciers particuliers, sont classés désormais dans le domaine périglaciaire, mais leur appellation dans les trois langues alpines, tout comme leur méconnaissance par le grand public, nous les fait conserver dans le champ d'investigation de cet article.

L'implication des autorités locales est importante car, comme dans tout projet de développement territorial, il est nécessaire que les élus, tout comme les habitants, s'approprient celui-ci. Dans ce but, la multifonctionnalité des espaces naturels doit bien être comprise et admise de tous, afin de préserver des dégradations les panneaux et autres aménagement qui seront postés sur le sentier. Parfois, ces mêmes autorités, propriétaires des lieux, peuvent prendre l'initiative de développer l'offre touristique mettant en valeur ses patrimoines.

\section{3 - Une diversification de l'offre touristique par les collectivités territoriales}

Une commune qui possède sur son territoire un glacier peut souhaiter en faire la promotion dans le but de diversifier son offre touristique, de prolonger la saison et ainsi se démarquer des territoires proches. En général, ces projets se réalisent dans le souci d'une mise en valeur qui cherche à minimiser l'impact touristique. Mais tel n'est pas toujours le cas, comme on peut l'observer pour le sentier glaciaire élaboré par la station de Zermatt en 2006. Ce sentier part du sommet du téléphérique de Trockener Steg à 2939 m et conduit en 23 arrêts à la station d'arrivée du Schwarzee para- dise à $2583 \mathrm{~m}$. C'est le seul sentier didactique sur les glaciers qui se parcourt en descendant! Les remontées mécaniques de Zermatt (Matterhorn glacier paradise) en partenariat avec l'office de tourisme de la commune qui l'ont créé mettent avant tout en valeur l'importance des aménagements réalisés sur le domaine skiable. L'enneigement artificiel qui couvre $60 \%$ des $183 \mathrm{~km}$ de pistes, assure la pratique du ski tout au long de l'année. Bien sûr, d'autres informations concernant l'environnement paraglaciaire, la faune ou la flore sont aussi abordées. Mais le traitement réservé au réchauffement actuel est particulièrement révélateur. Le retrait actuel du glacier est présenté comme une péripétie dans une histoire plurimillénaire des glaciers alpins. Aucune relation n'est faite avec le réchauffement global lié à l'impact des pratiques humaines : «... the fact that the Alpine glaciers are currently melting away, is nothing special, it is just part of a centuries long coming and going of snow and ice...» (...le fait que les glaciers alpins fondent actuellement n'a rien de particulier, c'est seulement une étape d'un processus centenaire d'avance et de recul de la neige et de la glace...). Les réchauffements passés sont présentés comme des périodes fastes de l'histoire de la région, le col du Théodule permettant alors le transit de nombreux voyageurs et marchandises qui ont fait la fortune de la vallée. L'histoire pluriséculaire du climat, présentée ici, n'est pas mise en perspective avec le réchauffement global actuel pour lequel aucune explication autre qu'une nouvelle péripétie naturelle n'est développée.

D'autres communes ont aussi développé ce type d'outil de valorisation patrimoniale, souvent incitées par les espaces protégés auxquels elles appartenaient. Ainsi, la commune de Champagny-le-haut a aménagé en 2008 un sentier glaciaire le long de la haute-vallée du Doron de Champagny au sein du Parc national de la Vanoise.

Si les objectifs de ces trois séries d'acteurs sont complémentaires, les stratégies ne sont pas toujours similaires. Par exemple, les sentiers promus par des centres de recherche font plus souvent l'objet d'une mise en valeur écrite, matérialisée par un support papier, qualifié par Daniel Jacobi d' «exotexte» (communication personnelle), alors que les communes ou les espaces protégés préfèrent souvent voir matérialisé sur le terrain le fruit de leurs efforts et bien sûr de leurs investissements, qualifié par le même auteur d'«endotexte».

\section{III - LES CARACTÉRISTIQUES SÉMIOTIQUES DES DIFFÉRENTS SENTIERS}

L'analyse qualitative des 30 sentiers inventoriés a permis d'établir une typologie en trois catégories clairement identifiées, qui ne se retrouvent pas toujours dans l'analyse des documents de médiation supports de ces sentiers. Ceci illustre, parfois, la mauvaise mise en synergie des objectifs des acteurs travaillant au projet, un sentier d'interprétation n'étant pas tout à fait un sentier de randonnée et les outils de médiation qui l'accompagne n'étant pas non plus des guides d'excursion de terrain. 


\section{1 - Le choix de l'itinéraire}

La majorité des sentiers proposés possèdent une dénivelée voisine de $500 \mathrm{~m}$, même si l'offre s'étend d'une dénivelée négative pour le sentier de Zermatt à plus de $1000 \mathrm{~m}$ pour le sentier du Gébroulaz en Savoie. (figure 5)

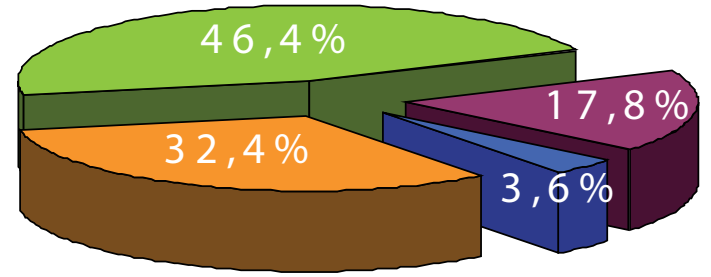

$\square$ Inf. à $0 \square$ Entre 0 et $300 \mathrm{~m} \square$ Entre 300 et $700 \mathrm{~m} \square$ Sup. à 700

Figure 5 - Répartition des sentiers d'interprétation glaciaire en fonction de leur dénivelée.

Cette répartition, mise à part l'exception du sentier de Zermatt, est à mettre en relation avec trois types de pratiques de randonnée. Les sentiers à la dénivelée inférieure à $300 \mathrm{~m}$ s'adressent à un public familial dans le cadre d'une balade qui, même avec des enfants ne dépassera pas une à deux heures. Entre 300 et $700 \mathrm{~m}$, on passe à une offre qui va permettre à la famille de partir à la journée, en pique-nique en montagne (photo 2), la durée du parcours sera alors de trois à cinq heures, ponctuée par les différents arrêts proposés le long du sentier qui devront donc combiner des points d'observation pertinents avec la nécessité de marquer des pauses régulières. Dans le dernier, cas le sentier s'adresse à un public sportif pour lequel l'aspect physique du parcours va être certainement tout aussi important que son aspect didactique. Si cette distinction en trois catégories des sentiers étudiés apparaît $a$ posteriori comme évidente, elle ne semble pas avoir forcément été réfléchie par les concepteurs a priori, puisque l'on s'aperçoit que certains fascicules, destinés à un très jeune public, sont associés à des sentiers ayant près de $800 \mathrm{~m}$ de dénivelée, comme c'est le cas avec le sentier de Champagny en Vanoise (Gotti, 2008).

En fonction de l'altitude de départ des sentiers, même ceux à dénivelée réduite peuvent impliquer la marche sur glacier et nécessiter donc un équipement adapté, comme dans le cas du sentier du centenaire sur le glacier Forni, en Lombardie.

Dans tous les cas, l'aménagement de ces sentiers dans des zones soumises au réchauffement climatique impose un entretien et une surveillance liée à l'impact de ce réchauffement (Pelfini, 2007). Le sentier du Centenaire, qui donne à découvrir le glacier Forni, le second plus important glacier d'Italie, a dû être fermé au cours de l'été 2005 en raison des conséquences du changement climatique sur sa moraine de rive droite. Celle-ci, qui possède un cœur de glace, subissait d'im- portantes déformations liées à la fonte de ce cœur, menaçant le déclenchement d'un glissement de terrain. L'instabilité créée dans cette moraine, après l'été caniculaire de 2003, a nécessité la fermeture du sentier par mesure de protection dans l'attente du réaménagement de cette portion et de sa réouverture quelques années après (Belo, 2006).

\section{2 - Les registres sémiotiques empruntés par les outils de médiation}

Panneaux, bornes et fascicules ou bandes dessinées sont autant d'outils de vulgarisation scientifique qui vont être développés au service des enjeux pédagogiques liés au sentier (figures 6 et 7). L'un des enjeux principaux est ici un transfert de connaissances et nous centrerons notre analyse sur l'ensemble des thèmes abordés par les différents supports récoltés, mais, afin de ne pas surcharger l'exposé, nous utiliserons une typologie tirée de la classification effectuée lors de l'analyse du panel.(tableau 1). Chaque sentier ne reprenant que certains thèmes présentés ci-dessus, on retrouve la typologie définie précédemment par l'analyse du jeu des acteurs à l'origine des créations. En effet, trois tendances peuvent être observées :

\section{a - Sentier militant}

L'aspect didactique a, dans ce cas, pour objectif une prise de conscience de la nécessité de protéger ces milieux, et l'environnement plus largement. Parmi les techniques pédagogiques utilisées dans ce cadre, les scénarios du futur sont des outils qui permettent de projeter les décisions d'aujourd'hui sur l'environnement de demain (figure 8). Ainsi, Max Maisch, qui a travaillé sur le sentier du glacier Morteratsch, propose deux scénarios d'évolution possible de la vallée lorsque le retrait glaciaire en aura libéré le fond : la poursuite du développement touristique avec l'urbanisation des espaces rendu vierges, ou bien la création d'un nouveau barrage en réponse à une pénurie d'eau certainement encore plus marquée dans le futur mais aussi dans le but de soutenir la production énergétique.

\section{b - Sentier éducatif}

La fonction principale de ce sentier est la transmission d'informations scientifiques concernant principalement les notions de base de géomorphologie. L'objectif est alors d'éduquer le touriste afin de le rendre capable d'interpréter d'autres paysages ou phénomènes d'origine glaciaire. Parmi les techniques pédagogiques utilisées dans ce cadre, la conduite d'un raisonnement inductif à partir d'une observation de terrain prépare la mise en place d'un raisonnement déductif ayant souvent recours au principe de l'actualisme (figure 9). 


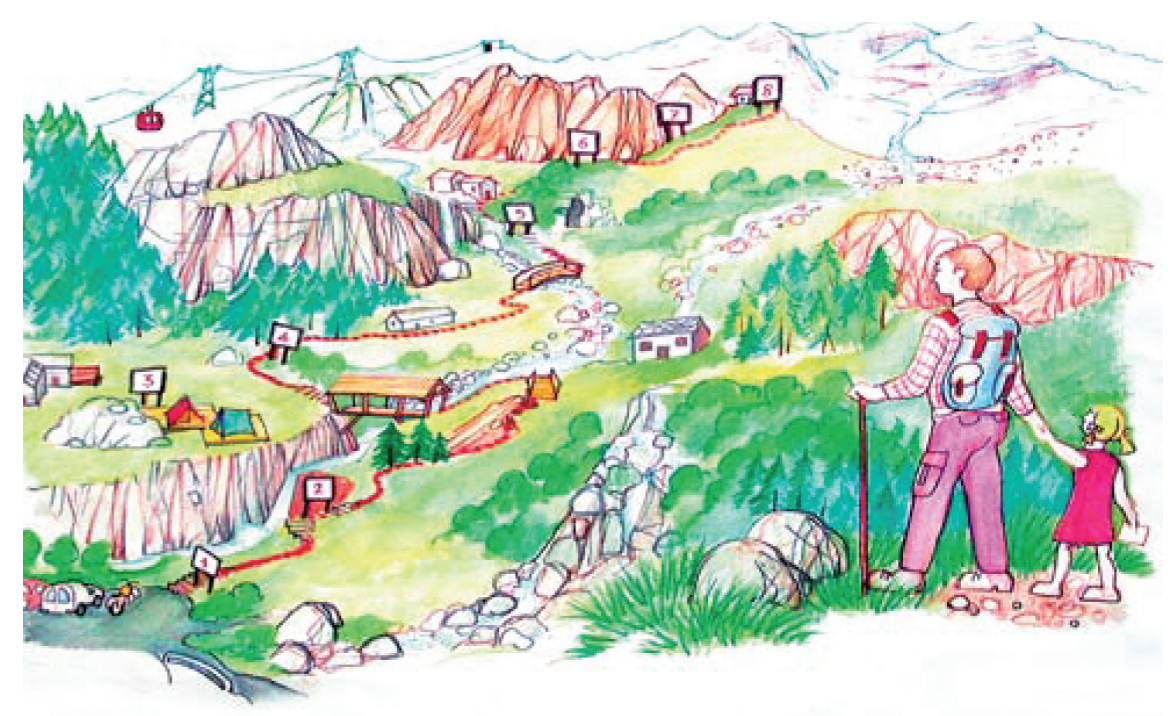

Figure 6-Itinéraire du sentier d'Alagna dans le parc naturel régional du Haut Val Sesia (source : fascicule d'interprétation $d u$ sentier).

Figure 7 - Itinéraire du sentier du glacier Pasterze dans le parc national des Hohe Tauern (sur photo aérienne et sur carte topographique; source : fascicule Gletscherweg Pasterze).
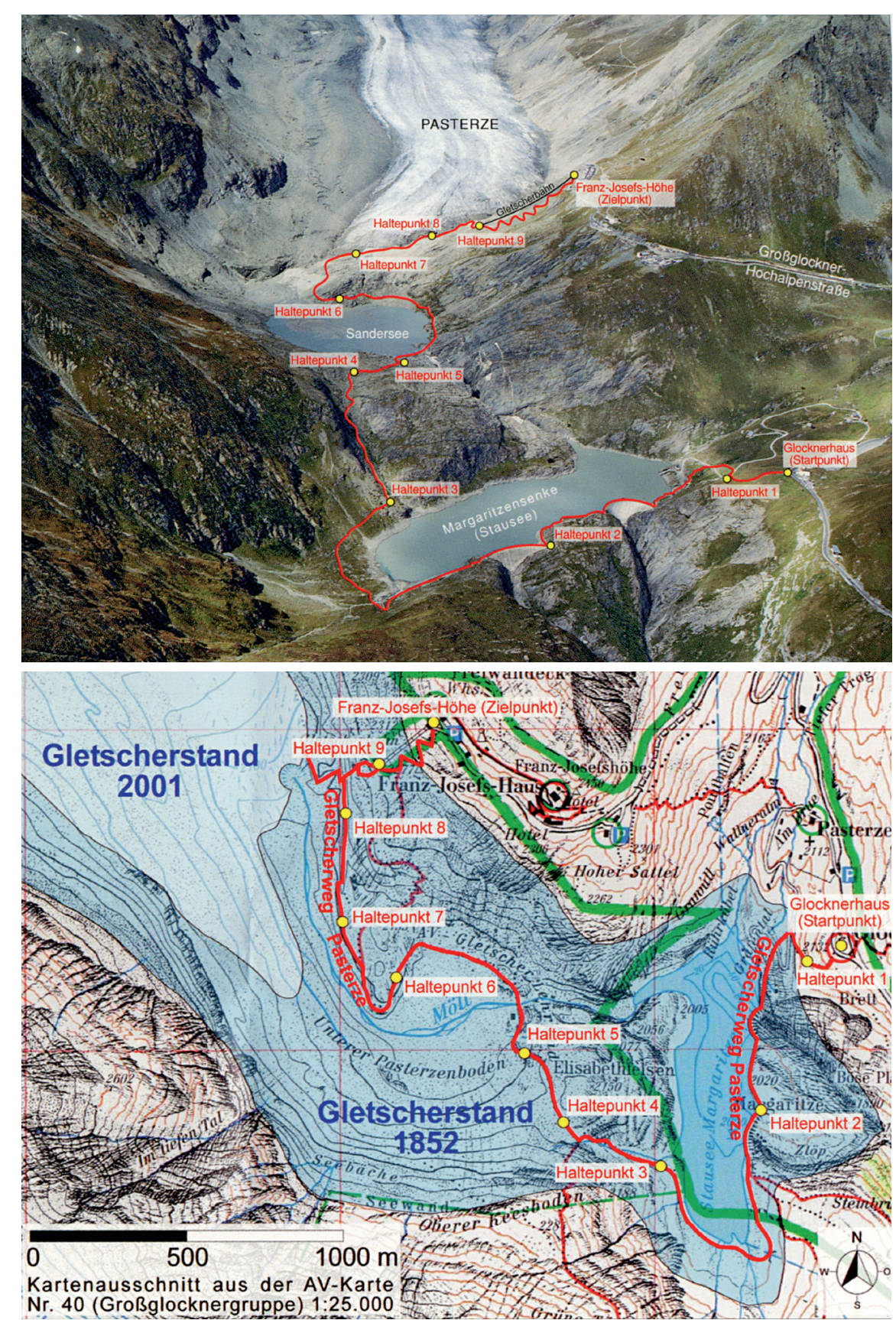


\begin{tabular}{|c|c|c|c|}
\hline \multirow[b]{2}{*}{ Thèmes abordés } & \multicolumn{3}{|c|}{ Nom du sentier } \\
\hline & $\begin{array}{l}\text { Lebendiges } \\
\text { Gletschervorfeld } \\
\text { (CH) }\end{array}$ & $\begin{array}{l}\text { Gletscherweg } \\
\text { Obersulzbachtal } \\
\text { (Au) }\end{array}$ & $\begin{array}{l}\text { Le sentier des glaciers } \\
\text { de Champagny-en- } \\
\text { Vanoise } \\
\text { (Fr) }\end{array}$ \\
\hline Les glaciers : formation, écoulement... & $x$ & $x$ & $x$ \\
\hline L'érosion glaciaire & $x$ & $x$ & $x$ \\
\hline Les dépôts glaciaires & $x$ & $x$ & $x$ \\
\hline Les marges proglaciaires & $\mathrm{X}$ & $\mathrm{X}$ & $x$ \\
\hline Les glaciations quaternaires & $x$ & $x$ & $x$ \\
\hline Les risques naturels liés aux glaciers & & & $X$ \\
\hline $\begin{array}{l}\text { Réchauffement global et impact sur les } \\
\text { glaciers }\end{array}$ & $X$ & & \\
\hline Géologie, faune, flore & $\mathrm{X}$ & $\mathrm{X}$ & \\
\hline L'homme et les glaciers & & & $X$ \\
\hline La recherche scientifique & $x$ & $x$ & \\
\hline Typologie & Sentier militant & Sentier éducatif & Sentier touristique \\
\hline
\end{tabular}

Tableau 1 - Liste des thèmes abordés par les différents types de sentiers.
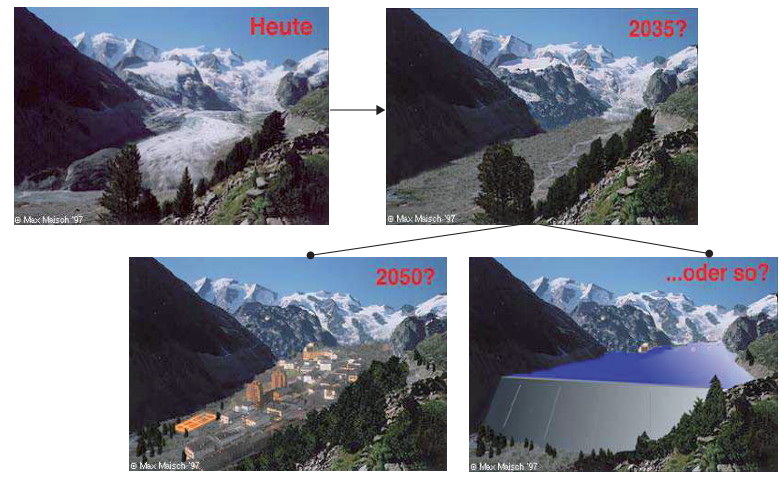

Figure 8-Scénarios du futur appliqué à la vallée de Pontresina dans les Grisons (source : Max Maisch, Geografisches Institut, Universität Zürich).

\section{c-Sentier touristique}

Le sentier est avant tout créé afin de mettre en valeur une ressource naturelle perçue comme patrimoniale par les habitants, les autorités locales... Cette réalisation sert alors à démarquer un territoire afin de mettre en valeur ses spécificités. Dans ce cadre, des recherches historiques minutieuses sont conduites afin de retrouver toutes les archives concernant le glacier et sa vallée. Une iconographie propre doit être inventoriée (dessins, aquarelles ou photographies), voire reconstituée par infographie a partir de cartes. Parfois, les démarches sont plus originales comme dans le cas du glacier de l'Obersulzbach où l'histoire récente du glacier, de sa vallée et même de l'Autriche est rappelée à travers la traduction d'une coupe dendrochronologique (figure 10).

Si nous avons voulu ici souligner les spécificités de chacune des pratiques, il est bien évident que dans la réalité celles-ci se conjuguent dans la plupart des

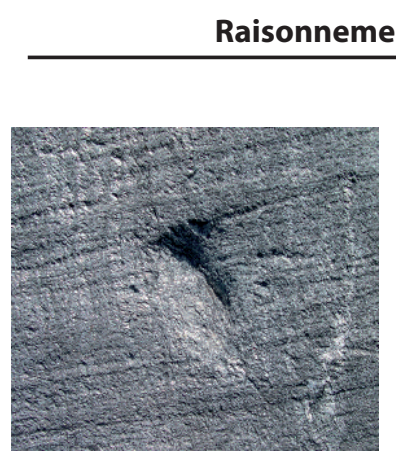

Observation de terrain : niche d'arrachement

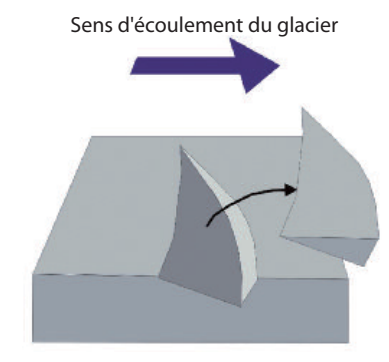

Schéma interprétatif

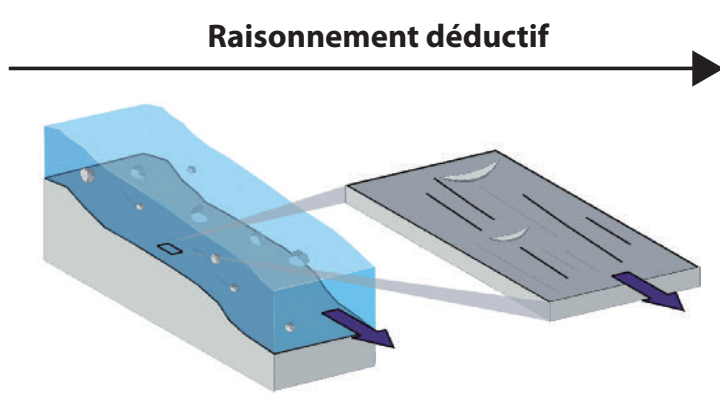

Application du principe de l'actualisme

Figure 9 - Cheminement pédagogique d'interprétation d'une niche d'arrachement (adapté du fascicule accompagnant le sentier de Zermatt).

fascicules et des panneaux étudiés. Il est important aussi que des thèmes secondaires soient abordés au cours de la promenade afin que l'ensemble des participants y trouvent un intérêt. Enfin, comme dans toute médiation scientifique, le concepteur doit susciter auprès de son lecteur trois types d'interactivité : 


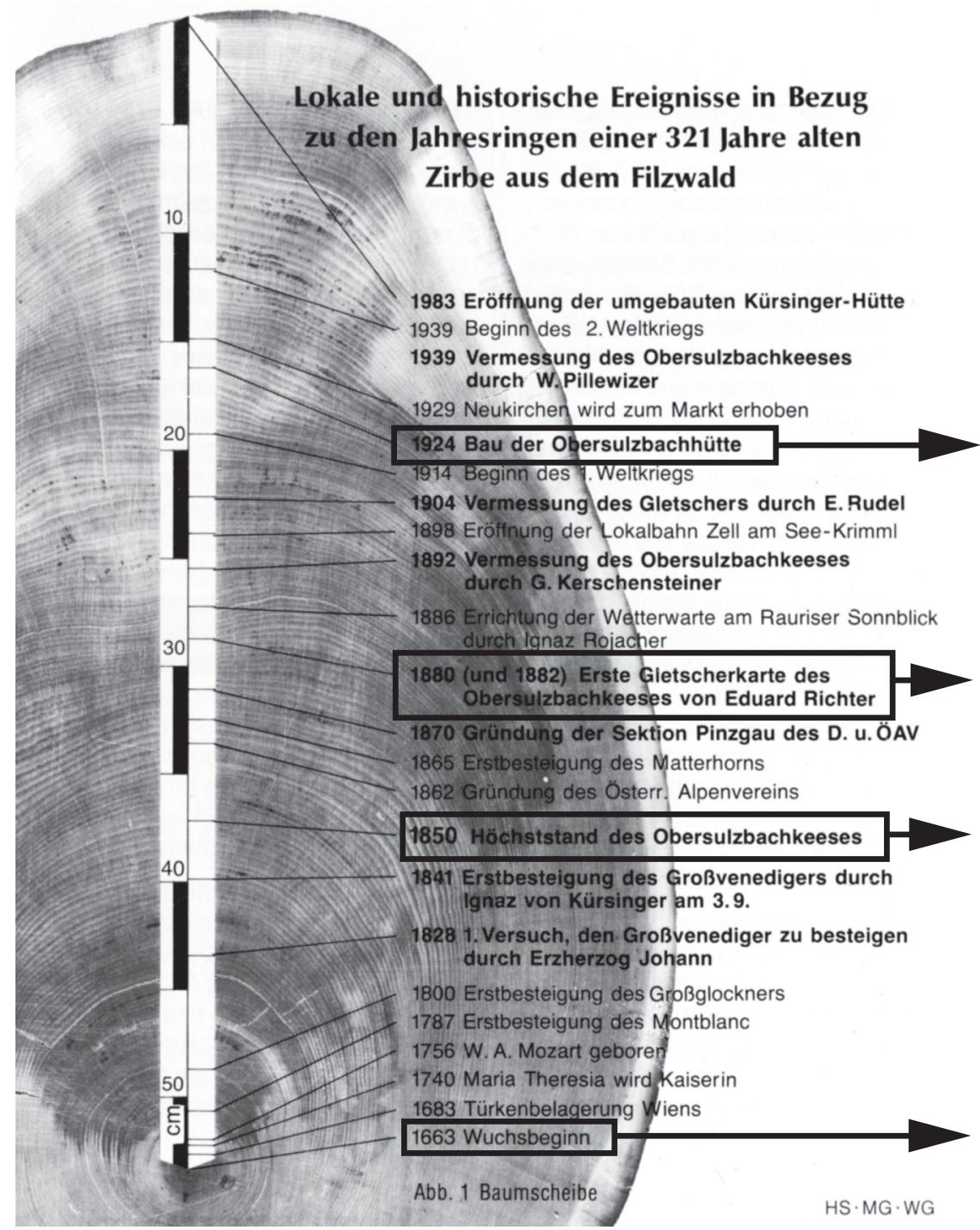

\section{Construction du refuge de l'Obersulzbach}

Première carte du glacier de I'Obersulzbach par Edouard Richter

\section{Maximum d'extension du glacier de l'Obersulzbach}

\section{Début de l'enregistrement des fluctuations du glacier}

Figure 10 - Présentation de l'histoire du glacier Obersulzbachkee par report des événements sur une coupe de tronc (source : Fascicule d'interprétation «Gletscherweg Obersulzbachtal»)

- une interactivité émotionnelle (notion clé de l'interprétation de Tilden) que les paysages glaciaires suscitent sans difficultés ;

- une interactivité manuelle avec le recours à de nombreuses observations et expérimentations de terrain qui peuvent être faites le long du parcours ;

- et enfin une interactivité intellectuelle, puisque le but de l'apprentissage d'un certain nombre de notions scientifiques ne doit pas être perdu de vue.

\section{Conclusion}

Le développement des sentiers d'interprétation à partir des années 1970, d'abord sur le territoire d'espaces naturels protégés, a permis de conjuguer les objectifs d'information et d'éducation à l'environnement avec l'essor des pratiques sportives de pleine nature. Dans les Alpes, 30 sentiers ont ainsi vu le jour sur le thème des glaciers, permettant une diversification de l'offre touristique dans le but de redynamiser le tourisme estival des hautes vallées.

Dans le cadre de ce travail, une typologie a pu être construite à partir de l'analyse quantitative et qualita- tive du corpus des sentiers retenus. Celle-ci illustre les jeux d'acteurs à l'origine des réalisations étudiées : des gestionnaires d'espaces protégés qui ont pour principal objectif l'éducation à l'environnement, mais aussi la prise de conscience d'une ressource naturelle en voie de disparition, des scientifiques qui contribuent ainsi à mieux faire connaître la géomorphologie en tant que champ disciplinaire à part entière, des acteurs du tourisme qui souhaitent mettre en valeur leur patrimoine au cœur d'une offre de plus en plus concurrentielle. 
Cette typologie forcément simplificatrice mériterait d'être interrogée plus en détail. L'étude comparative exhaustive des 30 supports de médiation réalisés permettrait ainsi, outre de définir le corpus sémantique et thématique adopté par chacun de ceux-ci, de mieux cerner les techniques didactiques utilisées : reformulation du discours scientifique, type de transposition, analyse scripto-visuelle... Il semble aussi nécessaire d'entreprendre un travail d'évaluation auprès des publics à qui sont destinés ces sentiers. Quelques tentatives ont déjà été faites dans d'autres pays (Kestler, 2005 ; Berrebi, 2006), mais en France ce type d'approche reste à développer afin d'améliorer l'offre et de mieux répondre aux attentes de ce public, un quatrième acteur... mais non des moindres puisque c'est à lui avant tout que l'offre est destinée.

\section{Remerciements}

Je tiens à remercier Micha Schlup, de l'Université de Lausanne, pour ses remarques concernant la station de Zermatt, Daniel Jacobi, professeur à l'Université d'Avignon, pour la relecture constructive, ainsi que mes collègues Fabien Hobléa et Véronique Peyrache-Gadeau qui ont bien voulu m'apporter leurs conseil dans la rédaction de ce travail.

\section{BIBLIOGRAPHIE}

Arsenault G., 2001. Développement et expérimentation d'un modèle d'interprétation pour éduquer les élèves de cinquième année au milieu dunaire. Mémoire de Maîtrise en Etudes de l'Environnement, Faculté des études supérieures, Centre Universitaire de Moncton, Québec, $129 \mathrm{p}$.

Astolfi J.-P., Develay M., 2005. La didactique des sciences. PUF éd., coll. Que sais-je ? $6^{\text {ème }}$ édition, 127 p.

Belo M., 2006. Ice core moraine collapse at Forni Glacier (Italian Alps): a case of tourist risk. Présentation orale, $10^{\text {ème }}$ rencontre glaciologique alpe, Munich, 2006.

Berrebi Y., 2006. Les sentiers didactiques. Analyse de la perception $\mathrm{du}$ public face à quatre réalisations géodidactiques. Mémoire de licence, Unil, Institut de Géographie, $200 \mathrm{p}$.

CAYla N., 2009. Glaciers actuels et géomorphologie paraglaciaire, quelques exemples de valorisation touristique au sein de l'arc alpin. Bulletin de l'Association de Géographes Français, 1, 96-109.

Dellantonio E., Roghi G., 2007. Sentiero geologico del Dos Capèl Der geologische des Dos Capèl ed APT Val di Fiemme, $40 \mathrm{p}$.

EDER R., 2007. Geologie am weg - Anregungen zur Gestaltung von geotrails. Abhanlungen der geologischen Bundesanstalt, 51, 29-34.

Garavaglia V., Pelfini M., 2008. Thematic mountain trails and dendrochronological fieldwork for a didactic approach related to glacier history, glacier geomorphosites and tree responses to global change. Geophysical Research Abstracts, 10.

Goтtr C. 2007. A la découverte des glaciers de Champagnyen-Vanoise. Parc National de la Vanoise, éd., 19 p.

Hose T, 2008. Towards a history of geotourism : définitions, antecedents and the future dans : The history of geoconservation. Geological society, special publication, 300, 37-60.

JACOBI D., 1999. La communication scientifique: discours, figures, modèles. Presses Universitaires de Grenoble, coll. Médias \& sociétés, 277 p.

KestLeR F., 2005. Der Tölzer Lobus des würmeiszeitlichen IsarLoisach-Gletschers als Gegenstand einer geodidaktischen Exkursion. Thèse Fakultät für Geowissenschaften der Ludwig-Maximilians de l'Université de Munich, 260 p.
MERCIER D., 2007. Le paraglaciaire, évolution d'un concept. In Du continent au bassin versant Hommage au professeur Alain Godard, 341-353.

Mose I., 2000. Hohe Tauern National park : a model for protected areas in the Alps. In Protected Areas and Regional Development in Europe, 99-113.

Nutz M., 2003. Blockgletscherweg Dösener Tal National park Hohe Tauern. Grazer Mitteilungen der Geographie und Raumforschung, 32, 1-5.

Pelfini M., Bossoni M., 2007. Un esempio delle interazioni fra dinamica geomorfologica e frequentazione turistica : la rapida evoluzione dei geomorfositi di alta montagna e l'incremento del rischio lungo gli itinerary glaciologici. In Geologi e Turismo, Atti del terzo congresso nazionale di Associazione geologia e turismo, 135-142.

Pirocchi A., 1998. Sentiero glaciologico al ghiacciaio d'Aurona. Parco Naturale Veglia e devero, 18 p.

Reynard E., 2004, La géomorphologie et la création des paysages. In Paysages géomorphologiques - Séminaire de troisième cycle de géographie CUSO, Travaux et Recherche, 27, 9-19.

Rohn-Brossard M., 2006. Parc naturel, chemin à thème et impact économique. Mémoire de fin d'étude de l'ECOFOC, Faculté des sciences naturelles, Université de Neuchâtel, 91 p.

Sellier D., 2009. La vulgarisation du patrimoine géomorphologique : objets, moyens et perspectives. Bulletin de l'Association de Géographes Français, 1, 67-81.

Smiraglia C., Diolaiuti G., Pelfini M., 2005. Valorizzazione e salvaguardia dell'alta montagna. I sentieri naturalistici : riflessioni teriche, realizzazioni, prosposte. In $\mathrm{La}$ valorizzazione turistica dello spazio fisico come via alla salvaguardia ambientale, Pàtron éd., Bologna, 303-315.

SLUPETZKI H., 1988. Gletscherweg Obersulzbachtal. Naturkundlicher Führer zum Nationalpark Hohe Tauern, Band 4, Oesterreichischer Alpenverein, $80 \mathrm{p}$.

TiLden F., 1957. Interpreting our heritage. Chapel Hill, The University of North Carolina press. 



\section{NEIGE et GLACE de MONTAGNE \\ Reconstitution, dynamique, pratiques}

\section{Sommaire}

Editorial

Introduction

\section{1 - Reconstitution}

Coutterand S. et al. - Le lobe glaciaire lyonnais au maximum würmien : glacier du Rhône ou/et glaciers savoyards ?

Ravanel L. et al. - Désenglacement du haut bassin versant du Vorz (massif de Belledonne, Isère), au Tardiglaciaire et à l'Holocène.

Rey P.-J. - Sociétés et fluctuations du climat dans les Alpes nord-occidentales au Néolithique moyen.

Le Roy et al. - Étude des fluctuations glaciaires du Petit Âge de Glace dans le Massif des Écrins : apports de la lichénometrie.

Kirkbride M.P. - Datation des moraines holocènes d'Islande par tephrochronologie : un état de l'art.

Le Roy et al. - La dendroglaciologie, ou l'apport de l'étude des cernes d'arbres pour la reconstitution des fluctuations glaciaires holocènes.

Rabatel A. - Évolution glaciaire dans les andes subtropicales chiliennes entre 1955 et 2007 : conséquences pour la ressource en eau.

Le Roy et al. - Un inventaire des aérophotographies du massif du Mont Blanc.

\section{2 - Dynamique}

Ravanel L. - Évolution géomorphologique de la haute montagne alpine dans le contexte actuel de réchauffement climatique.

Gruber S. - Le permafrost de haute montagne.

Deline P. et al. - L'Aiguille du Midi (massif du Mont Blanc) : un site remarquable pour l'étude du permafrost des parois d'altitude.

Saulnier G.-M. et al. - Un éléphant volant est-il un oiseau ? Perspectives pour l'observation hydrométéorologique des milieux de montagne.

Jobard S. - L'instrumentation du glacier du Baounet : quels apports pour la traçabilité des mesures environnementales ?

Moreau L. - L'exploration du cryokarst glaciaire et son intérêt scientifique pour l'étude du drainage des eaux de fonte.

Mazué R. et al. - Suivi de l'évolution de la couverture detritique d'un glacier noir par photo-comparaison : le glacier d'Estelette.

\section{3 - Pratiques}

Paccard P. - Réchauffement climatique et ressource neige en domaines skiables.

Gauchon C. - Les hivers sans neige et l'économie des sports d'hiver : un phénomène récurrent, une problématique toujours renouvelée.

Laslaz L. - L'exclusion des glaciers des zones centrales des Parcs nationaux de la Vanoise et des Écrins et leur équipement pour le ski d'été.

Cayla N. - Les sentiers d'interprétation glaciaire : des outils de valorisation différenciée des glaciers et de leur territoire.

Lambert R. - Cartozonage : de la carte au zonage du risque avalanche.

Moulin A. et al. - L'incertitude liée aux avalanches dans les Alpes du Nord : identifications et implications pour la gestion.

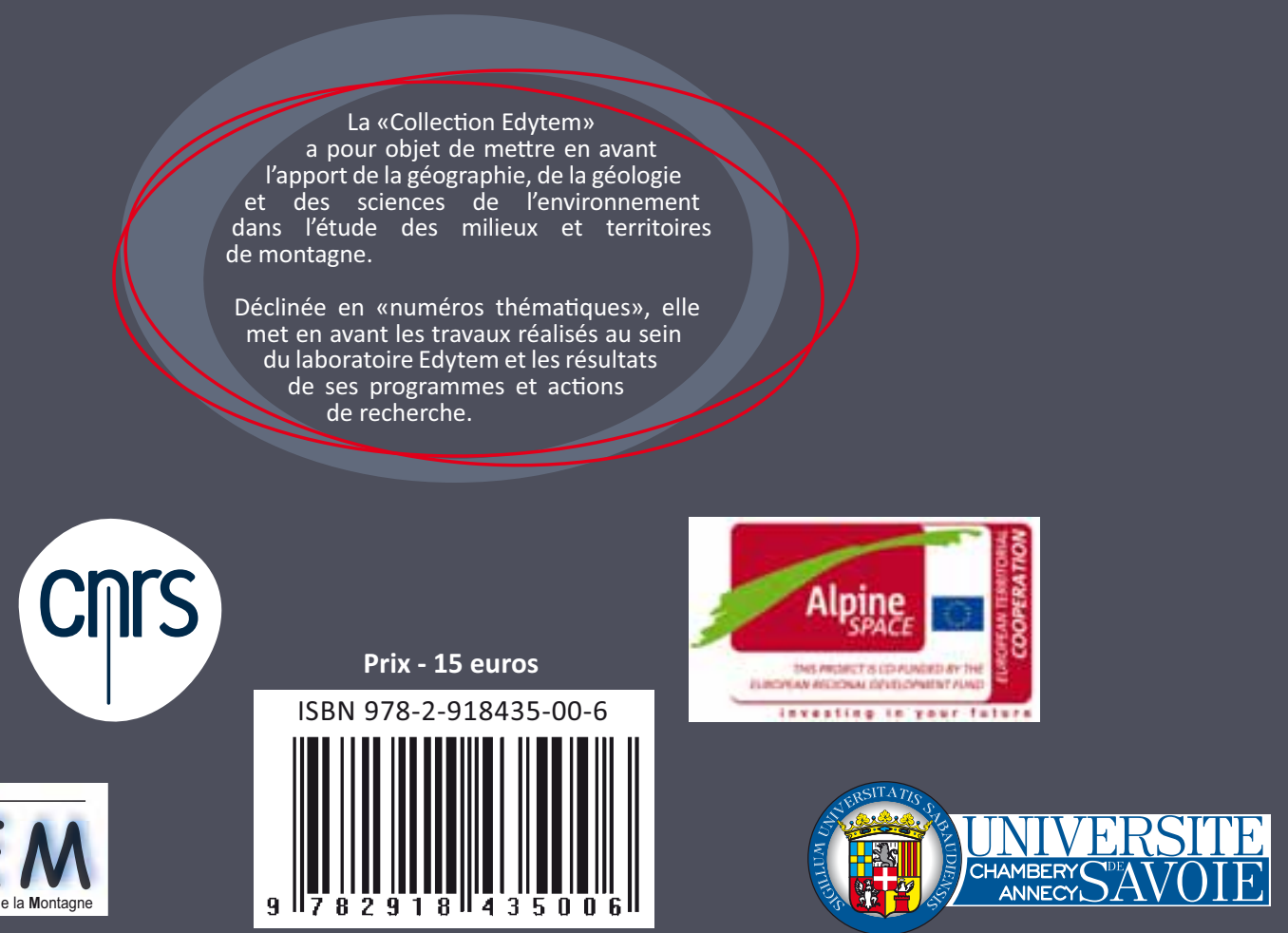

